\title{
3.7 Automated Scarfing Process for Bonded Composite Repairs
}

\author{
Dirk Holzhüter, Alexander Pototzky, Christian Hühne, \\ Michael Sinapius
}

\begin{abstract}
Today's bonded composite repairs rely heavily on manually grinding. The human influence on scarf tolerances and consequently on assembly and structural performance can be reduced by automating the process of scarf manufacturing. A process based on contact free surface scanning, surface reconstruction, automated repair design and automated milling of the repair scarf is presented. A machine and software design for validation purposes is described. Several repair specific design considerations relevant for the construction of a mobile scarfing machine are discussed. The redesign of a standard 3-axis milling machine to a mobile automated scarfing unit is presented and the architecture of the associated software framework described. An outlook to future validation steps is given.
\end{abstract}

\subsubsection{Motivation}

The steady increase of CFRP-Structures in modern aircrafts will reach a new dimension at the entry of the Boeing 787 into service. The extensive use of Carbon Fiber Reinforced Plastics (CFRP), e.g. in fuselage and wing shells, marks a major step forward in the application of modern composite materials. Those structures tend to be damaged more often compared to composite structures on classic aircrafts because of their location. Typical threats for those aircraft structures are environmental hazards like lightning and bird strikes, airport operations (e.g. service vehicles), maintenance and pilot handling (e.g. tail strike) [1].

Repairs of composite aircraft structures are already of great economic importance. Lufthansa Airlines carried out 1600 repairs on composite structures in 2006 (Fig. 1) [2]. A standard repair procedure on composite components is the bonded scarf patch repair [3]. The damage size will be determined by a NDT procedure, e.g. ultra sonic or thermography. The damage is commonly removed by hand drilling or grinding. The paint is locally removed down to the first ply. The typical procedure of preparing the bonding surface is to continuously scarf the structure around the damage in a circle (Fig. 3.7.1) or to manufacture a stepped contour [4]. Typical scarf ratios are in the range of 1:20 to 1:60 of the local structural thickness. The scarf is manufactured by manually grinding ply by ply by using an angular grind- 
ing tool. The craftsman orients oneself at the ply pattern to identify the local depth. A circular template is used to get the correct diameter. It is essential for the mechanical performance of the repair to fabricate the scarf in a small tolerance. The grinding activates the surface by removing the resin from the fibers and breaking open superficial chemical bonds for the following bonding process. The manufacture of the scarf can be quite complicated if the repair surface is curved or if local reinforcements, edges, thickness changes or bolted joints are located within the scarf area. Manually grinding a scarf is a slow process which can take several hours. The quality of the procedure also depends strongly on the experience of the technical personnel. Many different procedures are known for the patch manufacturing. The Co-Bonding of wet prepreg by using a film adhesive is a wide spread repair process among the industry. The wet lay up is still flexible and compensates even large tolerances. The curing temperature for the repair lay up as well as the adhesive is restricted to $130^{\circ} \mathrm{C}$. Local heat sinks on the parent structure restrict the use of higher ranges. The out-of-autoclave curing condition requires special repair prepregs. The alternative process of secondary bonding a cured patch requires a separate tooling. The tooling is directly moulded directly in the scarf by using a low temperature prepreg. This female tooling is moulded a second time to produce a male tool which is used in an autoclave to manufacture the proper patch at original processing conditions. Due to manufacturing distortions it is hardly possible to use a film adhesive for the bonding. Normally a paste adhesive is better suited to compensate the tolerances although both types of adhesives are used today. The process is much more time consuming compared to a wet-lay up process but produces a better mechanical performance. The cured patch repair is mainly restricted to military aircrafts while wet lay up is a preferred process for helicopters.

Up to now the bonded composite repair is a highly manual process. Especially the manufacturing of the scarf is a process with a huge human factor which may be minimized by the automation of this process step.

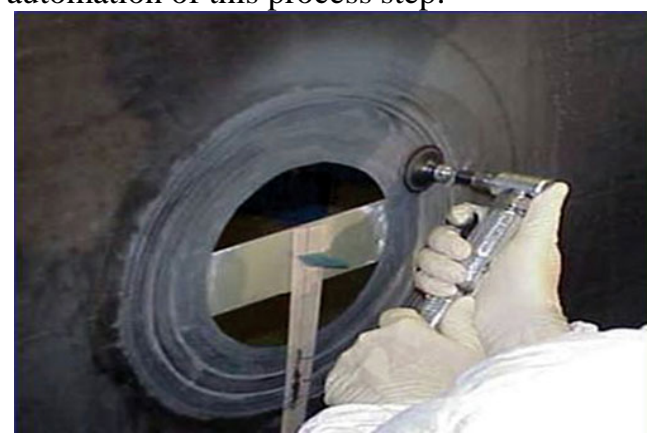

Fig. 3.7.1 Manual scarfing by angular grinding [5]. 


\subsubsection{The Automatic Scarfing Process}

The state of the art scarfing procedure has a great human impact on manufactured tolerances which influences the mechanical performance as well as assembly tolerances of the repair. The automation of this process is a major step forward in supporting the certification of bonded repairs for primary aircraft structures.

The manual grinding is therefore replaced by a numerical controlled (NC) milling of the scarf. A major requirement of the automatic milling process is the need for the local geometry information mainly the surface contour and thickness distribution. Baker [6] described in 2006 a CNC milling of a scarf contour. The surface geometry has been acquired by contacting the surface with a probe. The data was used afterwards for the CNC programming. In 2009 Wittingham [7] describes the usage of a surface profiling equipment and 5 axis milling to produce scarfs of repair specimen. Up to now no mobile process for the machining of 3D scarf repairs is known.

The proposed process chain automatic scarfing process starts by acquiring the geometry surrounding the repair (Fig. 3.7.1). The specified region surrounding the damage is scanned and local surface heights are saved to a point cloud data format. The data is automatically used to virtually reconstruct the surface by a NURBS (Non-Uniform B-Spline) approximation of the point cloud generating a repair surface for a CAD repair design (Fig. 3.7.2).. A software framework marshalling a CAD-kernel automatically generates a scarf design depending on user specific information like the scarf diameter, the scarf ratio, and the scarf depth (Fig. 3.7.3).. The design is directly based on the reconstructed surface. The CAD geometry may also be used in the future to generate cutting patterns for prepreg or wet layup patch repairs as well as for a finite element modelling of the repair. The CAD model is mainly used for outlining a milling strategy and to simulate the milling process offline (Fig. 3.7.4). A milling strategy is than simulated and compiled into a standard command code for CNC devices (G-Code). The generated command file is loaded into the control software of the mill and used for the machining process (Fig. 3.7.5). The milling of the scarf depends on the control commands which have been derived from the surface reconstruction. It is therefore essential that the machine does not move between the scanning process and the machining. The machine needs to be fixated directly onto the repair surface in order to assure this. This also solves problems with induced machining vibrations. Many rotorcrafts are equipped with rubber gears which tend to vibrate easily. A surface mounted solution is preferable in these cases. A mounting via suction cups is a common solution for surface mounted machines.

The number of needed control axis depends on the required repair geometry. A 3axis portal is only capable of machining a continuous scarf because of the curvature of the repair scarf. The direction of the machining tool cannot be normal to the surface which is necessary to machine the rims of a stepped repair. A 5-axis portal machine or a 7-axis serial robot would be necessary to manufacture both stepped and continuous scarfs which allow of aligning the tool normal to the surface. Experimental comparison between stepped and scarf repairs showed a supe- 
riority of the scarf repair in static tensile testing which supports the sufficiency of a 3-axis solution for future repair applications.
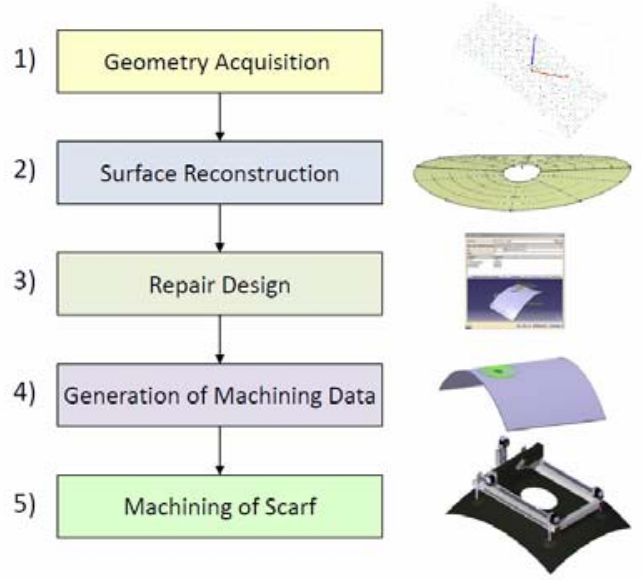

Fig. 3.7.2 Automatic scarfing process chain.

\subsubsection{Machine Design for the Automatic Scarfing Process}

The Automatic Scarf Concept has been realized in a specific machine design in order to validate concept assumptions and prove concept feasibility. Fig. 3.7.3 visualizes the realized machine which has been derived from the requirements. The basis of the machine is a customized 3-axis High-Z 720/T from CNC-STEP with a machining area of $720 \mathrm{~mm} \times 400 \mathrm{~mm} \times 110 \mathrm{~mm}$. The machining area has been chosen to manufacture typical scarf ratios of up to 1:40 in a 3mm laminate for an assumed damage of $50 \mathrm{~mm}$. The configuration is geometrically able to machine a continuous scarf but not a stepped one as outlined for 3-axis devices in the previous paragraph. Two stepping motors for the $\mathrm{x}$-axis, one for the $\mathrm{y}$-axis, and one for the z-axis each drive a recirculating ball screw with a feed rate of $0,00625 \mathrm{~mm}$ per $1 / 8$ step. The maximum motor rates have been experimentally tested to identify suitable machining speeds. The machining time is scaled by the feed rate linearly and by thickness and scarf ratio non-linearly. Fig. 3.7.4 visualizes the dependency of machining time on scarf ratio and thickness. The data has been generated by simulating the machining of different circular repair scarfs. Assuming a machining speed of $1200 \mathrm{~mm} / \mathrm{min}$ a standard repair with a scarf ratio of 1:20 and 3mm laminate thickness would be machined in 40 minutes. 


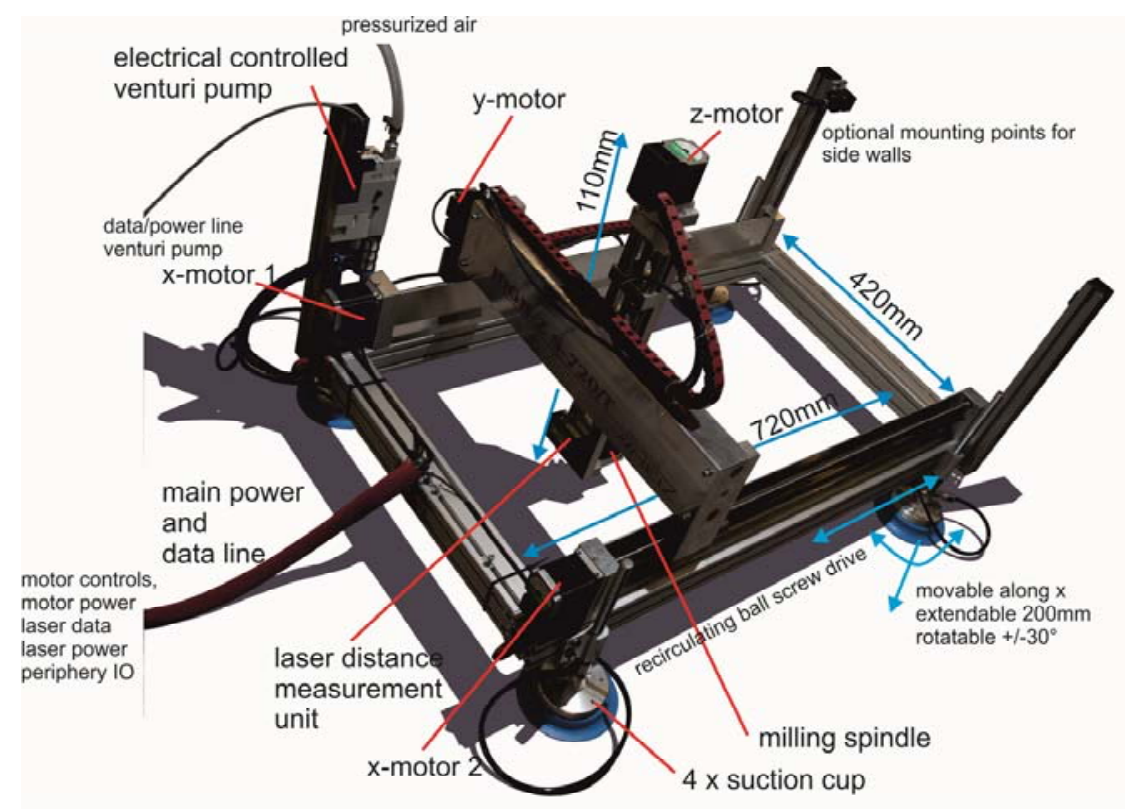

Fig. 3.7.3 Machine realized for validation of automated scarfing process.

The needed machining precision is derived from the tolerance of the scarf contour. A deviation of $\pm 0.1 \mathrm{~mm}$ of scarf contour results in a maximum failure of $4 \mathrm{~mm}$ in repair radius (assumed scarf ratio of 1:20). The maximum failure is scaled by scarf ratio linearly but not by thickness. This means in the given example case that the scarf ratio is reduced by $4 \%$ at maximum which seems still acceptable.

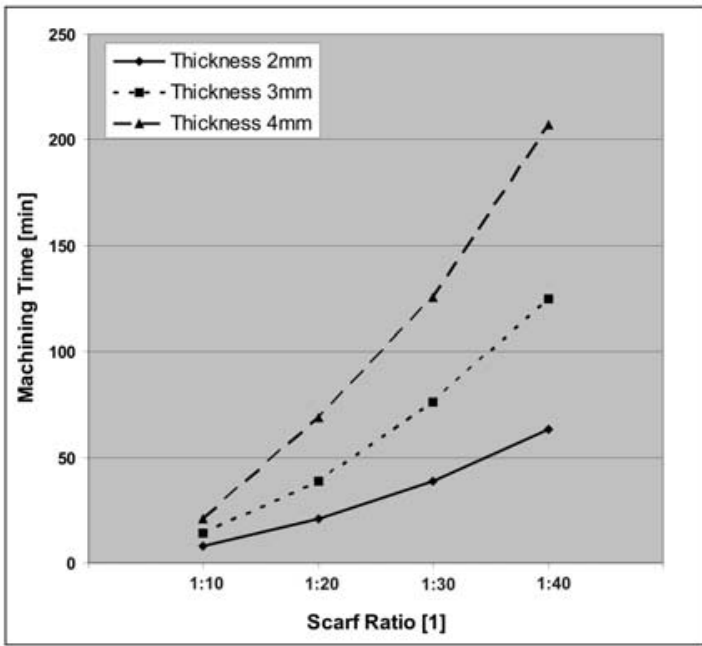

Fig. 3.7.4 Dependency of machining time of scarf ratio and structural thickness. 
The machines reproducible precision is specified by the manufacturer at $0.01 \mathrm{~mm}$. The milling spindle has standard rating of $1000 \mathrm{~W}$ and is able to cover turn rates of $5000 \mathrm{U} / \mathrm{min}$ up to $25000 \mathrm{U} / \mathrm{min}$. It holds tooling diameters of $1 \mathrm{~mm}$ to $8 \mathrm{~mm}$. The machine is controlled manually by software inputs or automatically by scripted commands (G-code). A separate hardware motor controller converts digital inputs into control signals for the stepping motors and is able to control all three axis simultaneously. The virgin machine weighs approximately $30 \mathrm{~kg}$.

The machines first modification is a $3 \mathrm{~mW} 660 \mathrm{~nm}$ wavelength laser distance measurement unit. The laser safety class is 3R which allows the usage of the laser without safety restrictions. It is directly mounted besides the milling spindle and aligned with the machines z-axis. It measures the relative height between the laser diode and a surface beneath. The maximum precision of the laser is specified at $\pm 0.03 \mathrm{~mm}$. Experimental validation showed a maximum error of $\pm 0.02 \mathrm{~mm}$ by analyzing 400 measurements. The laser system is synchronized with the step signals of the machines $\mathrm{x}$-motors by the synchronization unit and is DC-isolated by the isolation unit. The synchronization unit consists mainly of an integrated controller (IC) equipped with an internal counter. The IC can be programmed to trigger a laser measurement at a specific step count of the x-axis. Data communication is handled by RS232 serial protocol and transmitted via a hardware FTDI ${ }^{\odot}$ RS232/USB converter to a PC. The synchronization allows the calculation of the local $\mathrm{x}$ and $\mathrm{y}$ position of the machine if proper zeroing of the machine axis has been done. The point laser combined with the hardware provides a very simple hardware implementation to measure a local surface contour and synchronize this with the actual machining position. The scanning is restricted to line patterns due to the fact that the stepping and directional signals of the $\mathrm{x}$-axis motor controller is solely used to trigger a measurement. The offset between milling tool and laser is measured and compensated before machining. The position of a small step in a template tool is measured and touched by the tool allowing a maximum precision of approximately $0,08 \mathrm{~mm}$.

The second main modification is the replacement of the standard mounting points by four suction cups. An electrical controlled venturi pump is operated by pressurized air and generates an absolute pressure of 0.1bar. Pressurized air is chosen as the main vacuum source because it is commonly available in repair shops or easily produced by portable compressor. Each suction cup withstands a shear force of $\sim 90 \mathrm{~kg}$ which is more than the machines total weight. The pump was chosen because of its fail safe design. An internal valve closes in case of pressure or electrical loss which locks the suction cups for a sufficient amount of time to secure machine and operating personnel. Each suction cup adjusts to a local structural curvature of about $\pm 15^{\circ}$. The mounting points are rotatable for about $\pm 30^{\circ}$ and movable in $\mathrm{z}$ and $\mathrm{x}$ direction. The system has been tested on different composite surfaces (typical peel ply and tooling surfaces) and different types of sandpaper granularity. Results are given in Fig. 3.7.5. 


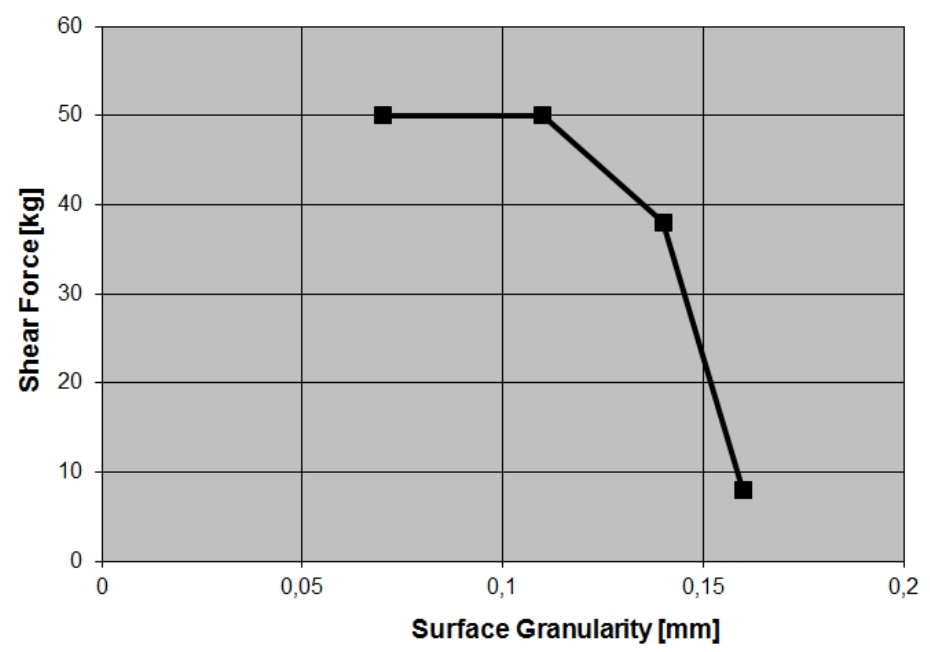

Fig. 3.7.5 Shear force of one suction cup for different surface granularities.

The third modification is a complete rewiring of the electrical interfaces. Fig. 3.7.6 shows the schematic of the electrical design. The machine has been divided into two units, the machining unit and the control unit. Both units are connected with only one electrical and one pressure line allowing a fast disconnect of the machine. A relocation test has been performed in approximately $15 \mathrm{~min}$. The electrical line contains the motor controller wiring, laser power and data transfer, and periphery I/O lines. All commands and data input/outputs are controlled from a portable PC. Everything has been mounted into a movable board for laboratory use only.

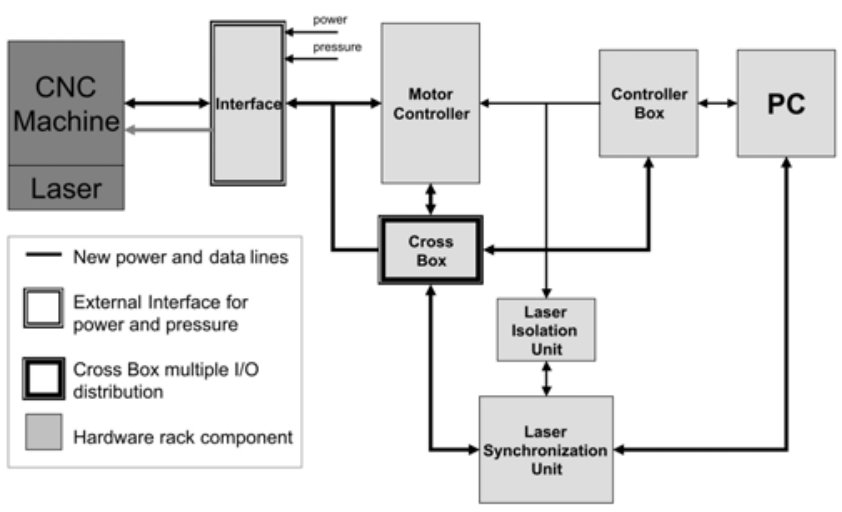

Fig. 3.7.6 Schematic of redesigned electrics. 


\subsubsection{Software Framework for the Automated Scarfing Concept}

A major aim of the automated scarfing concept is keeping the process simple in order to prevent damages to structure and personnel. This is the topmost priority of the developed software framework for command and control of the machining process. Fig. 3.7.7 shows the frameworks schematic. The software is developed in Microsoft Visual C\# object oriented programming which allows easy driver integration for periphery devices like the laser unit and the venturi pump. The whole software process has been split into multiple software modules to keep the software flexible for future developments.

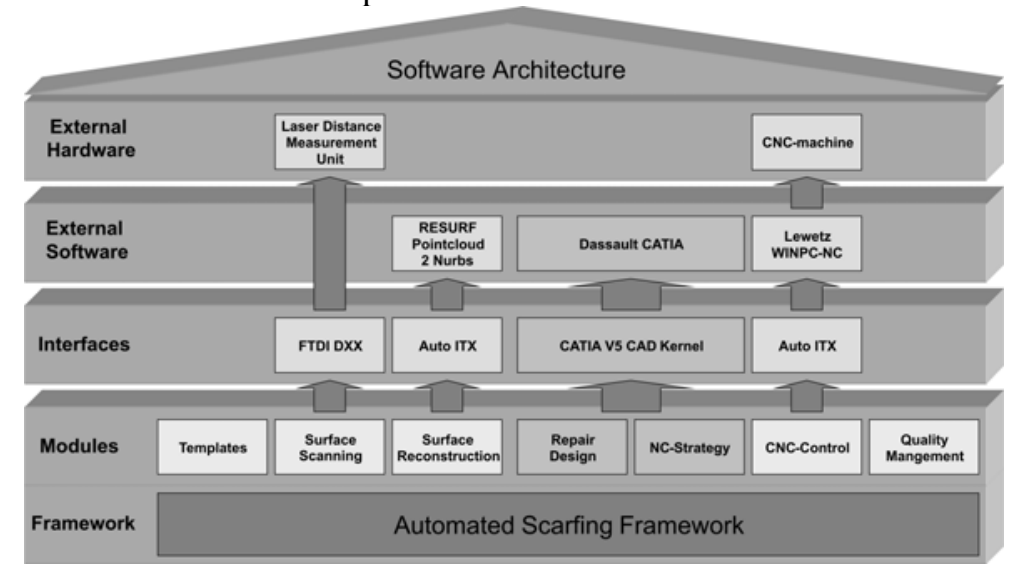

Fig. 3.7.7 Software schematic.

The template module allows the choice and definition of standard repair scenarios. This includes repair e.g. geometry and machining strategy. This allows standardizing the process and reducing human influence due to erroneous inputs. The second module controls the scanning process of the repair surface. The user is able to vary the scanning resolution in machines $\mathrm{x}$ and $\mathrm{y}$ direction. The software communicates with the laser via a $\mathrm{FTDI}^{\odot}$ USB/RS232 interface driver. It also generates a suitable G-code command script for the scanning process which is automatically loaded by the machines control program. The machine scans the repair surface in lines and returns the local $\mathrm{z}$ values of the laser which is saved in xyz-ascii data file. The data is processed by the surface reconstruction module. A proper 3D modelling of the repair design needs a G0, G1 and G2 continuous surface. Due to the noise in the laser measurement it is necessary to do a surface approximation. A 3D NURBS fitting of the point data was chosen. The algorithm for this fitting is implemented by using a $3^{\text {rd }}$ party software (Pointcloud2NURBS ${ }^{\odot}$ by Resurf ${ }^{\complement}$ ) which is a low cost tool. The output is an .igs data file. The software is marshalled via the AutoITX $^{\odot}$ interface driver allowing an easy integration of the reconstruction software. The repair design module controls the construction parameters of the repair design. It is possible to generate circular as well as elliptical scarf designs. Scarf ratios and repair diameters are used to virtually construct the repair design based 
on the reconstructed surface. The framework uses Dassault CATIA ${ }^{\odot}$ V5R18 via its API (Application Programming Interface). CATIA ${ }^{\odot}$ is a standard tool within aerospace industry and provides the geometry operations to construct the repair design. A cost efficient alternative is the OPENCASCADE ${ }^{\mathcal{O}}$ geometry library which is a freeware tool. Fig. 3.7.8 shows the design of the scarf contour. The inner and outer repair contours are defined in the machines xy-plane and projected (1) along the machines z-axis onto a tangential repair plane. These projections are wrapped over the repair surface (2) and projected in local normal direction (4) onto offset surfaces (3) to construct local support points. These support points are connected by a spline function to define the 2D scarf contour (5). This scheme is used to design a spline every $30^{\circ}$. These splines are used as guides to loft the scarf surface. The repair design is a parametric script which allows changing of scarf ratio, repair diameters and layout (circular or elliptic). For load optimized repair designs it is possible to define varying scarf ratios for the elliptic layout.

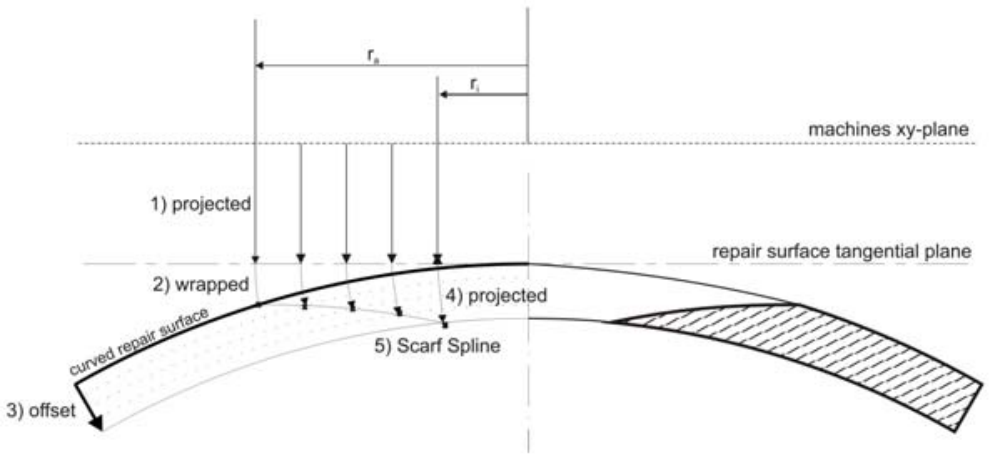

Fig. 3.7.8 Design of curved scarf contour.

Module number five is used define machining specific parameters and to generate the machining code. Especially tool diameters, machining feed and turn rates can be chosen. Additionally the machining strategy can be changed. NC-strategy influences scarf tolerances due to the fact that local laminate stresses relax during machining. In most cases a machining on nominal value without any roughening is chosen to prevent the part from deforming. This strategy wears the tool and introduces great machining forces. The module provides options to choose the standard or alternative strategies e.g. separate roughening and finishing. The sixth module controls the command software of the milling machine. This is done again via AutoITX. The milling process can be started and stopped from this module. The last module supports quality management processes. The geometry data is saved and a report is generated. The report contains a summary of manufacturing parameters and chosen options.

The developed modular software framework provides a command and control environment for the complete machining and design process of a 3D curved repair scarf. 


\subsubsection{Summary and Outlook}

The bonded composite repair process depends on the quality of the repair scarf. The automation of the repair scarf manufacturing is an important step forward in improving repair quality and reproducibility. A process based on contact free laser scanning, surface reconstruction, CAD-design and mobile CNC-milling has been developed and realized in an integrated machine design. The machine as well as the software design has been outlined. The validation of the concept still remains open for the future. Especially comparing manually manufactured scarfs to the automatically manufactured ones is the next step in validating the concept. The machine has been designed to achieve the necessary repair tolerances. This has to be demonstrated in different typical repair situations, e.g. circular repairs of curved panels. The process gives a new design freedom to the repair engineer. Especially load optimized repairs with varying scarf ratios or freeform scarf contours will be possible. Both options may improve robustness of the repair design and further improve mechanical performance of the bonded repairs.

A major future aspect to consider is the influence of grinding on the bonding characteristic of the surface. For certifying an automatic milling process as bonded repair preparation it is necessary to prove that the milled surface has the same bonding performance as a grinded one. A manual grinding after the milling would negate every advantage of the automatic scarfing because it would be done manually. Therefore it is essential to provide sufficient data to show compliance of bonding characteristics between grinded and milled repair surfaces. 


\section{References}

[1] Armstrong, K.B., L.G. Bevan, and W.F. Cole, Care and Repair of Advanced Composites2005: SAE International.

[2] Sauer, C., Lufthansa Perspective on Applications \& Field Experiences for Composite Airframe Structures, in 3rd FAA Workshop for Composite Damage Tolerance \& Maintenance2009, Lufthansa Technik: Tokyo, Japan.

[3] Baker, A.A., et al., Composite materials for aircraft structures2004: American Institute of Aeronautics and Astronautics.

[4] Niu, M.C.-Y., Composite Airframe Structures : Practical Design Information and Data 1st ed1992, Hong Kong: Hong Kong Conmilit Press. 664.

[5] Boeing, Boeing Patch Repair, SB09_compositesthematerials_i.jpg, Editor 2009, http://www.compositesworld.com/articles/composites-repair. p. A repair technician scarfs the area around a damage cutout prior to application of a composite patch. .

[6] Baker, A., S. Defence, and D.I.V. Technology Organisation Victoria Air Vehicles. Development of a Hard-Patch Approach for Scarf Repair of Composite Structure. 2006; Available from: http://handle.dtic.mil/100.2/ADA458447.

[7] Whittingham, B., et al., Micrographic studies on adhesively bonded scarf repairs to thick composite aircraft structure. Composites Part A: Applied Science and Manufacturing, 2009. 40(9): p. 1419-1432. 